\title{
On the LFM signal improvement by piecewise vibrational resonance using a new spectral amplification factor
}

ISSN 1751-9675

Received on 22nd November 2017 Revised 27th April 2018

Accepted on 29th May 2018 E-First on 10th October 2018 doi: 10.1049/iet-spr.2018.5101 www.ietdl.org

\author{
Pengxiang Jia ${ }^{1}$, Jianhua Yang1,2,3凶, Xin Zhang ${ }^{4}$, Miguel A.F. Sanjuánn ${ }^{5,6,7}$ \\ ${ }^{1}$ School of Mechatronic Engineering, China University of Mining and Technology, Xuzhou, 221116, People's Republic of China \\ ${ }^{2}$ Department of Mechanical Engineering, University of Michigan, Ann Arbor, MI, 48109, USA \\ 3 Jiangsu Key Laboratory of Mine Mechanical and Electrical Equipment, China University of Mining and Technology, Xuzhou, 221116, People's \\ Republic of China \\ ${ }^{4}$ School of Mines, China University of Mining and Technology, Xuzhou, 221116, People's Republic of China \\ ${ }^{5}$ Nonlinear Dynamics, Chaos and Complex Systems Group, Departamento de Física, Universidad Rey Juan Carlos, Tulipán s/n, 28933, \\ Móstoles, Madrid, Spain \\ ${ }^{6}$ Department of Applied Informatics, Kaunas University of Technology, Studentu, 50-407, Kaunas, LT-51368, Lithuania \\ ${ }^{7}$ Institute for Physical Science and Technology, University of Maryland, College Park, Maryland, 20742, USA \\ 凶-mail: jianhuayang@cumt.edu.cn
}

\begin{abstract}
The authors construct the piecewise vibrational resonance (VR) method by introducing the piecewise idea and combine the re-scaled and twice sampling methods for the linear frequency modulated (LFM) signal. Importantly, they put forward a new spectral amplification factor to quantify the VR. The new spectral amplification factor has some advantages as compared with the cross-correlated coefficient, which can also be used to quantify the aperiodic VR. The top value of the spectrum amplification factor is the necessary and sufficient condition for the occurrence of the optimal VR phenomenon. Here, they discuss the effects of different parameters on VR by using some numerical examples. The method described in this study provides an effective way to improve the LFM signal, and even other kinds of frequency modulated signals.
\end{abstract}

\section{Introduction}

The vibrational resonance (VR) is a resonance phenomenon first proposed by Landa and McClintock [1]. VR is similar in some sense to the well-known stochastic resonance (SR) phenomenon [2]. In the VR mechanism, the system is excited by two signals. One of them varies slowly with time, while the other signal varies faster with time. The slow varying signal is usually weak. By adjusting the amplitude of the faster varying signal, the weak slow varying signal can be amplified when VR occurs. In fact, according to the nonlinear dynamics theory, the fast varying signal plays a crucial role in the system parameters [3]. It can soften the stiffness of the nonlinear system. In other words, the parameters of the equivalent system depend on the fast varying signal. By adjusting the amplitude of the fast varying signal, the equilibrium(s) of the equivalent system will be changed. Then, the resonance may occur in the slowly varying signal, leading to the amplification of the weak slow varying signal. If the two excited signals are in harmonic form, the analytical results of the VR can be obtained by the method of direct separation of fast and slow motions [4, 5]. To our knowledge, VR has been investigated or applied in delayed systems [6-8], fractional systems [9-11], laser systems [12, 13], neuron systems [14-18], plant ecosystems [19], plasma systems [20], ferroelectric liquid crystals [21] etc. Besides investigations on abundant dynamical behaviours, VR can also be applied in the engineering field. For example, VR has been successfully applied in fault feature extraction in mechanical bearing systems [22]. VR is very similar but much simpler than SR. As we know, the SR is applied in various engineering fields. Hence, we predict that the VR has potential engineering applications in many fields.

In most investigations on VR, the excitations are usually constituted by a low-frequency harmonic signal and a highfrequency harmonic signal. Besides, the excitations can also be constituted by an aperiodic signal and a harmonic signal. The aperiodic VR, when the aperiodic signal appears in an aperiodic binary signal form, has been investigated in [23]. However, besides the aperiodic binary signal form, there are many aperiodic signals in other forms, which are also very important. Thus, we believe that it is very necessary to investigate VR when the excitations contain other kinds of aperiodic signals. Among the aperiodic signals, the frequency modulated signal is widely used in various research fields such as in radar signal processing [24-27], ultrasonics $[28,29]$, communications [30] etc. The linear frequency modulated (LFM) signal is a typical frequency modulated signal. Lin et al. make the SR occur in the LFM signal excited system based on the fractional Fourier transformation technology [31-33]. However, the fractional Fourier transformation is not a simple method. Moreover, comparing SR with the VR, the noise in SR is replaced by another determined auxiliary signal. It leads to the conclusion that VR is much easier to control and analyse. Thus, we believe that it is very necessary to investigate VR on the LFM signal improvement problem. Since the frequency of the LFM signal varies with time, it is difficult to make VR appear by using the traditional method. Hence, developing a new VR method to improve the LFM signal is our main motivation in this study.

The rest of this paper is outlined as follows. In Section 2, the piecewise idea and re-scaled transformation are introduced. We propose the new spectrum amplification factor in this section. Then, the VR can be realised by the piecewise re-scaled method. In Section 3, the piecewise twice sampling VR method is briefly presented. In Section 4, the main conclusions of the paper are given.

\section{Piecewise re-scaled VR}

We apply a classic bistable system to process the LFM signal

$$
\frac{\mathrm{d} x(t)}{\mathrm{d} t}=a x(t)-b x^{3}(t)+u(t)+F(t)
$$

where $a>0$ and $b>0$ are the system parameters. We consider the bistable system as a signal processor. $x(t)$ is the system variable. $u(t)$ is the LFM signal. $F(t)$ is the auxiliary signal which is used to 

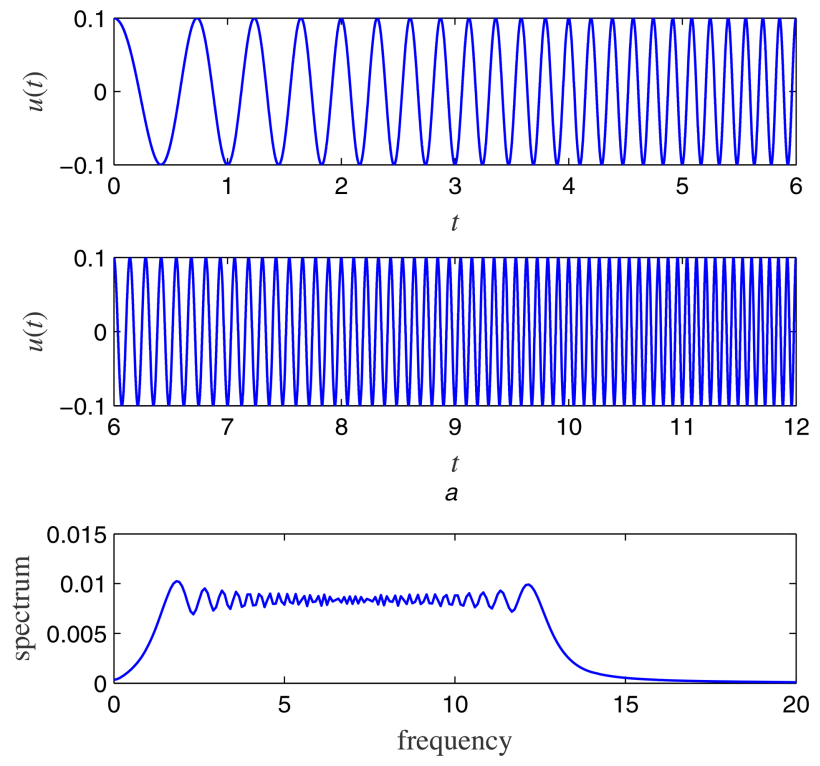

$b$

Fig. 1 The input LFM signal

(a) Time series of the LFM signal, (b) Spectrum of the LFM signal. The signal parameters are $A=0.1, \gamma=1$ and $f=1$

induce the VR phenomenon. Specifically, the LFM signal is given by

$$
u(t)=A \cos \left(\pi \gamma t^{2}+2 \pi f t+\phi\right),
$$

where $A, \gamma, f$ and $\phi$ are the amplitude, the chirp rate, the starting frequency and the initial phase, respectively. For simplification, we let $\phi=0$ all throughout the paper. It should be pointed out that the transient vibration frequency $f_{\text {in }}(t)$ of the LFM signal is

$$
f_{\text {in }}(t)=f+\gamma t .
$$

$F(t)$ can take different forms. Here, we construct the auxiliary signal in the following form:

$$
F(t)=B \cos \left(\pi k \gamma t^{2}+2 k \pi f t\right)
$$

where $B$ is the amplitude of the signal and $k>0$ is a positive constant.

To understand the LFM signal further, the time series and the corresponding spectrum are shown in Fig. 1. In Fig. 1b, the continuous spectrum of the LFM signal is clearly observed. It is clear that the spectrum of the LFM signal is different from that of the harmonic signal distinctly.

In a two harmonic signal excited system, the VR phenomenon can be quantified by the response amplitude which is calculated by the Fourier coefficients. With regard to the LFM signal-excited system, such as (1), the response amplitude is invalid. This is because the LFM signal does not have the discrete spectrum. To solve this problem, we propose here a new index, which is named as the spectrum amplification factor of the LFM signal. Specifically, we use $\eta$ to label it and let

$$
\begin{aligned}
& \eta=\frac{\frac{1}{f_{\text {final }}-f_{\text {initial }}} \int_{f_{\text {initial }}}^{f_{\text {final }}} S_{x}\left(f_{\text {in }}\right) \mathrm{d} f_{\text {in }}}{\frac{1}{f_{\text {final }}-f_{\text {initial }}} \int_{f_{\text {inital }}}^{f_{\text {final }}} S_{u}\left(f_{\text {in }}\right) \mathrm{d} f_{\text {in }}} \\
& =\frac{\sum_{i=1}^{n} S_{x}(i)}{\sum_{i=1}^{n} S_{u}(i)},
\end{aligned}
$$

where $S_{x}(\bullet)$ and $S_{u}(\bullet)$ are the discrete spectrum of the system output and the input LFM signal, respectively. The subscript $i$ means the $i$ th point in the discrete spectrum. There are $n$ points in total in the discrete spectrum. The integration variable $f_{\text {in }}$ is the transient vibration frequency which is defined in (3). Here, $f_{\text {initial }}$ and $f_{\text {final }}$ mean the transient vibration frequency at the start time and the end time of the time series. Substituting $t=t_{\text {inital }}$ and $t=t_{\text {final }}$ into (3), they can be obtained directly. By the indicator in (5), the amplification of the signal by the cooperation of the nonlinear system and the auxiliary signal is described explicitly. The spectrum amplification factor gives amplification degree of the LFM signal from an average viewpoint. When $\eta>1$ the LFM signal is amplified by the VR method. Furthermore, we will have much more excellent amplification if we have a larger value of $\eta$. The indicator in (5) is different from the former indicators such as the response amplitude or the cross-correlation coefficient used to quantify the VR or aperiodic VR phenomenon. In fact, as to the LFM signal, we can also use the cross-correlation coefficient to quantify the VR phenomenon. To show the superiority of our spectrum amplification indicator, we also use the cross-correlation coefficient as a comparison. The cross-correlation coefficient $C_{u x}$ between the input LFM signal and the output can be calculated by using the following formula:

$$
C_{u x}=\frac{\left.\sum_{j=1}^{m} u(j)-\bar{u}\right][x(j)-\bar{x}]}{\sqrt{\sum_{[u(j)-\bar{u}][x(j)-\bar{x}]}^{2} \sum_{j=1}^{m}}},
$$

where $\bar{u}$ and $\bar{x}$ are the average of the input LFM signal and the output, respectively.

To achieve a good match between the LFM signal and the system parameters, the re-scaled method is used here. This method has been used in the harmonic signal excited system successfully $[10,34,35]$. Moreover, due to the modulation of the frequency of the LFM signal, we introduce the piecewise idea to get a better matching. As to the considered signal, we divided it into several segmentations equally according to the total time of the signal. We use the same value of $B$ to obtain the output induced by each segmentation, respectively. Then, we join all segmentations in turn and calculate the quantitative indicator. Corresponding to each segmentation, we adopt a certain re-scaled parameter $\beta$. Specifically, $\beta$ is defined by

$$
\beta=\beta_{0}\left(f+\gamma t_{e i}\right),
$$

where $\beta_{0}$ is a constant and $t_{e i}$ is the end time of the considered segmentation. The process of the piecewise re-scaled method is as follows.

We let

$$
\tau=\beta t, \quad x(t)=z(\tau) .
$$

Then, (1) turns to

$$
\begin{aligned}
\frac{\mathrm{d} z(\tau)}{\mathrm{d} \tau}= & \frac{a}{\beta} z(\tau)-\frac{b}{\beta} z^{3}(\tau) \\
& +\frac{1}{\beta} u\left(\frac{\tau}{\beta}\right)+\frac{1}{\beta} F\left(\frac{\tau}{\beta}\right) .
\end{aligned}
$$

Letting

$$
a_{1}=\frac{a}{\beta}, \quad b_{1}=\frac{b}{\beta},
$$

then, (9) changes to

$$
\begin{aligned}
\frac{\mathrm{d} z(\tau)}{\mathrm{d} \tau}= & a_{1} z(\tau)-b_{1} z^{3}(\tau) \\
& +\frac{1}{\beta} u\left(\frac{\tau}{\beta}\right)+\frac{1}{\beta} F\left(\frac{\tau}{\beta}\right) .
\end{aligned}
$$

Recovering the signal to the original amplitude, we have 


$$
\begin{aligned}
\frac{\mathrm{d} z(\tau)}{\mathrm{d} \tau}= & a_{1} z(\tau)-b_{1} z^{3}(\tau) \\
& +u\left(\frac{\tau}{\beta}\right)+F\left(\frac{\tau}{\beta}\right) .
\end{aligned}
$$

As a result, the VR can be achieved by the following equation:

$$
\begin{aligned}
\frac{\mathrm{d} z(t)}{\mathrm{d} t}= & a_{1} \beta z(t)-b_{1} \beta z^{3}(t) \\
& +\beta u(t)+\beta F(t) .
\end{aligned}
$$

To observe the VR phenomenon, in Fig. 2, we adopt the spectrum amplification factor and the cross-correlated coefficient to get the optimal VR effect, respectively. In Fig. $2 a$, the optimal VR is achieved at the top value of $\eta$, i.e. the resonance peak of the curve. The critical value of $B$ corresponding to the peak is $B=0.42$. In Fig. $2 b$, the optimal VR is achieved at the resonance peak of the curve, herein, $B=0.46$. However, it is not the top value of $C_{u x}$ at the resonance peak in Fig. $2 b$. In other words, when the crosscorrelated coefficient achieves a large value, the VR phenomenon may not occur. In fact, the cross-correlated coefficient only labels the similarity between two different time series. The top value of the cross-correlated coefficient is the necessary but not the sufficient condition for the VR phenomenon. However, the top value of the spectrum amplification factor is the sufficient and necessary condition for the VR phenomenon. Actually, the spectrum amplification factor is superior to the cross-correlated coefficient. For example, if we adopt an optimisation algorithm to achieve the optimal VR effect, the spectrum amplification factor is a better objective function than the cross-correlated coefficient due to the optimal VR occurring as the sole top value of the spectrum amplification factor.

To show the VR phenomenon explicitly, in Fig. 3, we give the time series when $B$ makes $\eta$ achieve the peak in Fig. $2 a$. Apparently, the main components of the frequency are kept and their amplitudes are amplified. As a result, by our piecewise VR method, the LFM signal is improved excellently.

The frequency of the auxiliary signal influences the VR effect, as shown in Fig. 4. With the increase of $k$, the transient frequency of the auxiliary signal also increases. It leads to the decrease of the spectrum amplification factor. Certainly, according to the VR theory, the transient frequency of the auxiliary signal must be far larger than the corresponding transient frequency of the LFM signal, i.e. $k \gg 1$.

In Fig. $5 a$, the effect of the constant $\beta_{0}$ on the spectral amplification factor is shown. With the increase of $\beta_{0}$, the top value of $\eta$ becomes larger. This is because $\beta_{0}$ determines the re-scaled parameter $\beta$. Furthermore, a larger $\beta$ value makes the excitation to have a lower transient frequency in the equivalent system, (12). According to the nonlinear dynamics theory, an excitation with a lower frequency is easy to induce a stronger response. As a result, we have the result shown in Fig. $5 a$. To demonstrate the validity of the method, in Fig. 5b, we have chosen different parameters of the LFM signal. Apparently, VR occurs in all cases. This indicates that the validity of the method is independent of the signal parameters.

In all aforementioned figures, all calculations are based on the piecewise idea. The amplitude of the auxiliary signal used in different segmentations has the same value. We collect all outputs induced by different input segmentations as the whole output to calculate the quantified indicators $\eta$ and $C_{u x}$. When the considered indicator achieves the resonance peak, the whole output achieves the optimal VR effect. However, based on this idea, every segmentation of the output may not have achieved the optimal VR. This is because we may need different values of $B$ to induce the optimal VR in different segmentations. To clarify this confusion, we have Fig. 6, in which the $\eta-B$ curve is obtained separately for each segmentation. We find that different segmentations achieve the resonance peak almost at the identical value of $B$. This indicates that the whole output achieves the optimal VR, and also can make each segmentation to achieve the optimal VR approximately. Comparing Fig. 6 with Fig. $2 a$, there is no difference in nature between these two figures. Lines 1-4 are
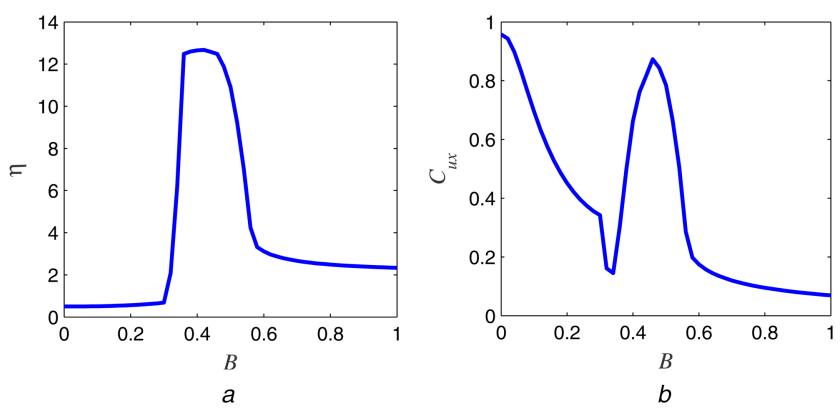

Fig. 2 The indices of the output

(a) Spectrum amplification factor $\eta$ versus amplitude $B$ of the auxiliary signal, (b) Cross-correlated coefficient $C_{u x}$ versus amplitude $B$ of the auxiliary signal. The simulation parameters are $A=0.1, \gamma=1, f=1, a_{1}=1, b_{1}=1, k=10$ and $\beta_{0}=300$
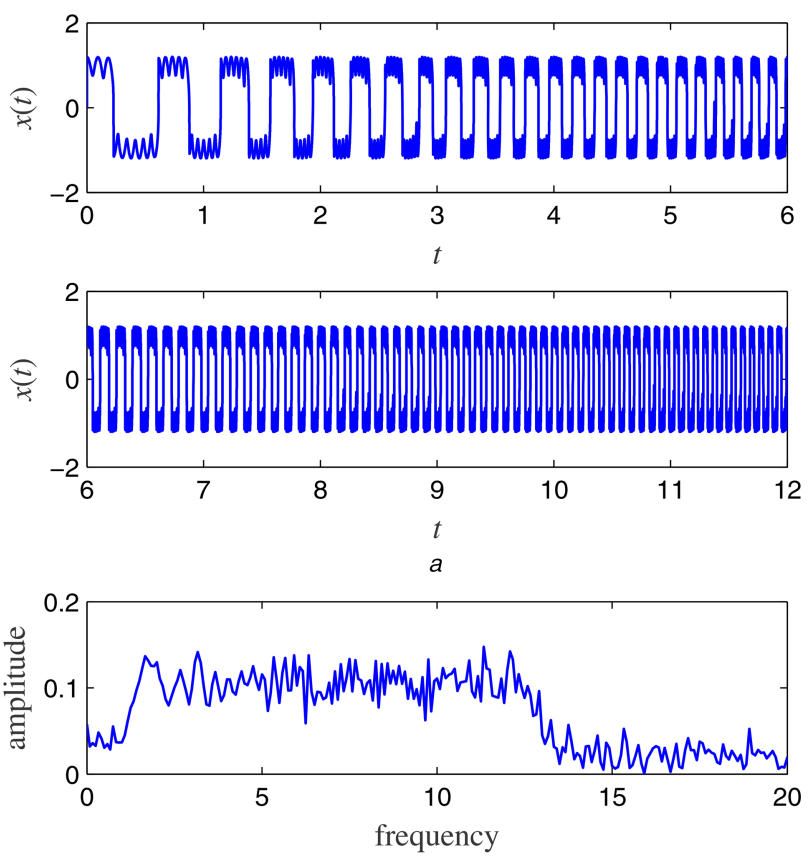

$b$

Fig. 3 The optimal output of the system

(a) Time series of the output corresponding to the optimal VR effect, (b) Spectral of the output corresponding to the optimal VR effect. The simulation parameters are $A=0.1, \gamma=1, f=1, a_{1}=1, b_{1}=1, B=0.42, k=10$ and $\beta_{0}=300$

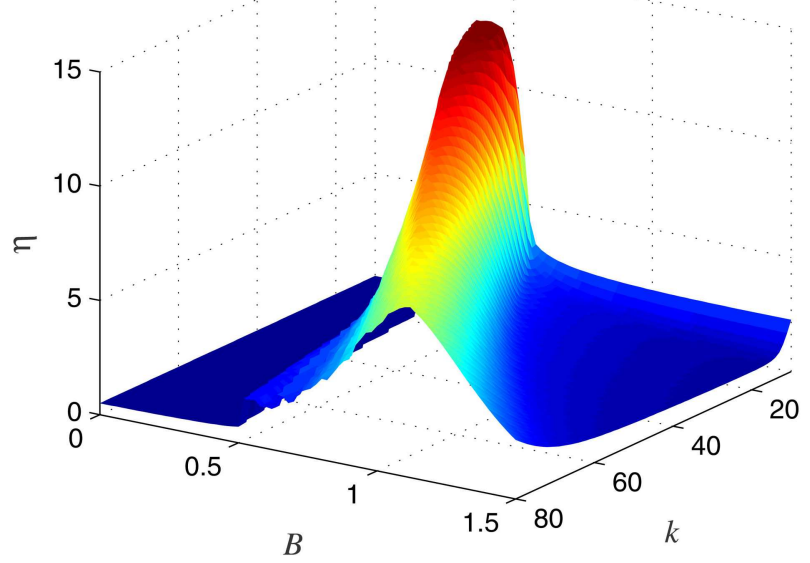

Fig. 4 Spectrum amplification factor $\eta$ versus the amplitude $B$ and the constant $k$. The simulation parameters are $A=0.1, \gamma=1, f=1, a_{1}=1$, $b_{1}=1$ and $\beta_{0}=300$ 

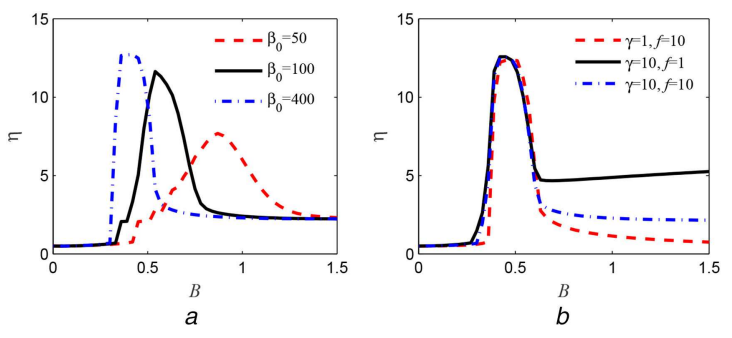

Fig. 5 Spectrum amplification factor $\eta$ versus the amplitude $B$ of the auxiliary signal, $A=0.1, a_{1}=1, b_{1}=1, k=10$, in (a) $\gamma=1$ and $f=1$, (b) $\beta_{0}=200$

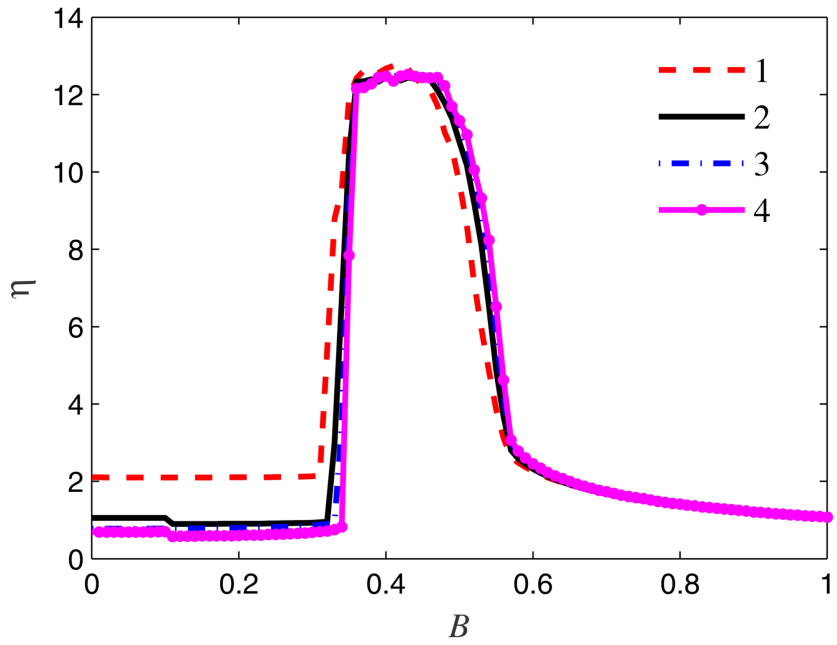

Fig. 6 Spectrum amplification factor $\eta$ versus the amplitude $B$ of the auxiliary signal. The simulation parameters are $A=0.1, \gamma=1, f=1$, $a_{1}=1, b_{1}=1, k=10$ and $\beta_{0}=300$. Lines $1-4$ are corresponding to the output induced by segmentations $1-4$, respectively

corresponding to the output induced by segmentations $1-4$, respectively. In lines $1-4$, the critical value of $B$ at the resonance peak is $B=0.41,0.4,0.43$ and 0.43 in turn.

Corresponding to the results in Fig. 6, the optimal VR output time series is shown in Fig. 7. Comparing Fig. 7 with Fig. 3, there is no natural difference between them yet.

Besides the auxiliary signal in (4), it can also be chosen in other forms, such as

$$
F(t)=B \cos [k(f+p \gamma) t]
$$

or

$$
F(t)=\underset{\{}{B \operatorname{sgn}} \cos [k(f+p \gamma) t]\},
$$

where $\operatorname{sgn}(\bullet)$ is the sign function. $p$ is a large constant and we let $p=12$ in the following analysis. In Figs. $8 a$ and $b$, we use the signal in (14) and (15) as the auxiliary signal, respectively. The VR effect is obvious in Fig. 8. Different forms of the auxiliary signal can induce VR by the piecewise re-scaled method. The form of the auxiliary signal has a trivial influence on the VR effect.

\section{Piecewise twice sampling VR}

The twice sampling method is another approach to deal with the high-frequency signal [36, 37]. Here, we combine the piecewise idea and the twice sampling method to put forward the piecewise twice sampling VR method. The procedure is as follows:

i. We divide the LFM signal into several segmentations equally in the time domain.

ii. We use the first sampling frequency $f_{\mathrm{s}}$ to sample each signal segmentation.
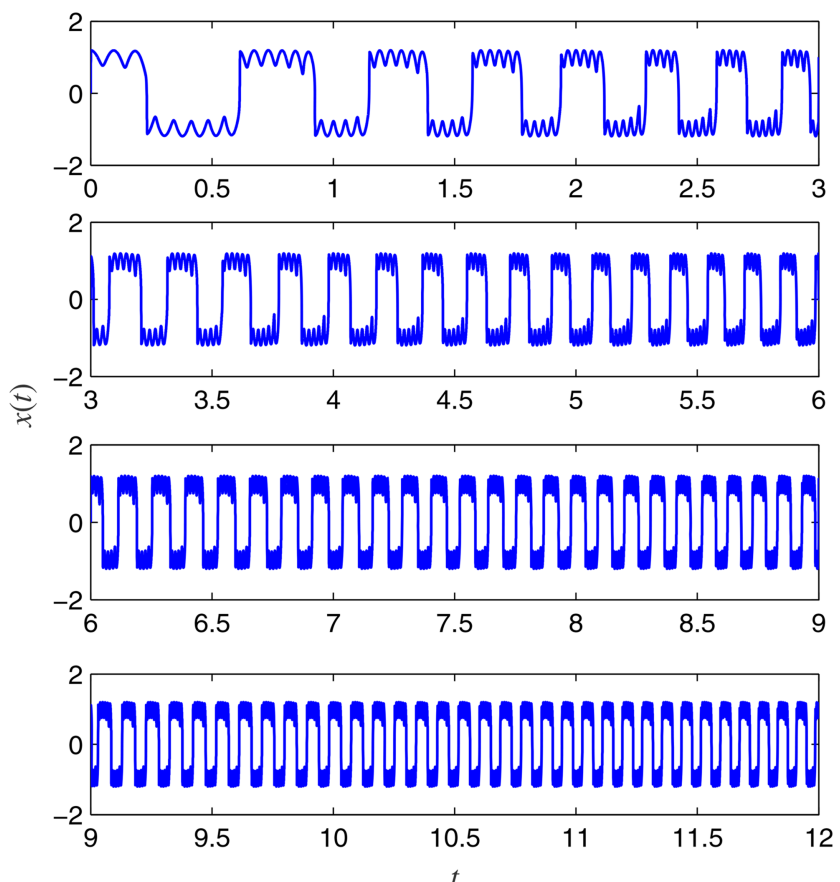

Fig. 7 Time series of the output corresponding to the optimal VR effect. The simulation parameters are $A=0.1, \gamma=1, f=1, a_{1}=1, b_{1}=1$, $k=10, \beta_{0}=300$, and from top to bottom $B=0.41,0.4,0.43$ and 0.43 , respectively
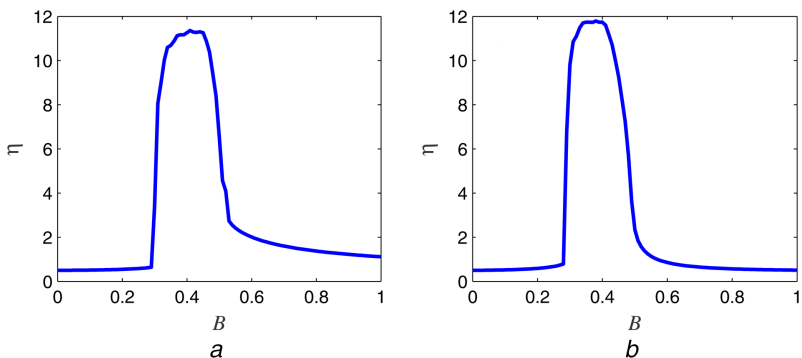

Fig. 8 Spectrum amplification factor $\eta$ versus the amplitude $B$ of the auxiliary signal

(a) (14) is used as the auxiliary signal, (b) (15) is used as the auxiliary signal. The simulation parameters are $A=0.1, \gamma=1, f=1, a_{1}=1, b_{1}=1, k=10$, and $\beta_{0}=300$

iii. For each sampled signal segmentation in the previous step, we use the twice sampling frequency $f_{\mathrm{s}} / m_{\mathrm{r}}$ to re-sample the signal segmentation. In other words, each signal segmentation is reconstructed. Through this operation, the transient frequency of the reconstructed signal will be $1 / m_{\mathrm{r}}$ times of the corresponding frequency of the original signal segmentation. We name $m_{\mathrm{r}}$ as the frequency reduced ratio. Here, $m_{\mathrm{r}}$ is defined by

$$
m_{\mathrm{r}}=m_{0}\left(f+t_{e i}\right),
$$

where $m_{0}$ is a constant and $t_{e i}$ is the time at the endpoint of the considered signal segmentation.

iv. The reconstructed signal segmentation is the input of the original nonlinear system. The output time series induced by each input signal segmentation is obtained. Then, we collect all the output time series in turn to construct a whole output.

v. The whole output is recovered to the first sampling frequency $f_{\mathrm{s}}$ according to the frequency reduced ratio $m_{\mathrm{r}}$.

vi. The indicator such as $\eta$ or $C_{u x}$ is calculated based on the reconstructed output in the last step.

Based on procedures (1)-(6), the piecewise twice sampling VR method is used to obtain Fig. 9. We use different values of $m_{0}$ in Fig. $9 a$. For larger values of $m_{0}$, the spectral amplification factor 

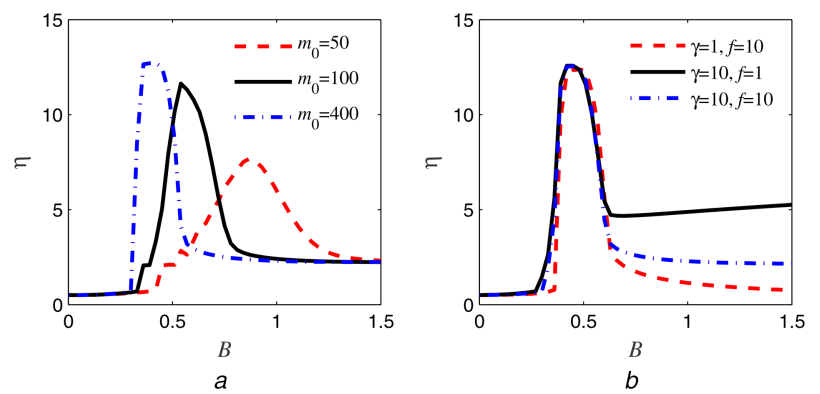

Fig. 9 Spectrum amplification factor $\eta$ versus the amplitude $B$ of the auxiliary signal, $A=0.1, a=1, b=1, k=10$, in

(a) $\gamma=1$ and $f=1$, (b) $m_{0}=200$

has a larger maximal value. In other words, larger values can induce stronger VR. This is because a larger value can make the twice sampled signal to have a lower transient frequency. Hence, it can induce a stronger response. In fact, the constant $m_{0}$ has the same effect as that of $\beta_{0}$ in the last section. In Fig. $9 b$, we use different parameters of the LFM signal. Although it results in different waveforms of the LFM signal, the VR effect still is present excellently. Comparing Fig. 9 with Fig. 5, we find that both the piecewise re-scaled VR method and the piecewise twice sampling method can achieve the same goal, i.e. realising an excellent VR phenomenon.

\section{Conclusion}

In the present work, the piecewise VR method is used to realise VR in the LFM signal excited system. Specifically, the piecewise rescaled VR and the piecewise twice sampling VR are used, respectively. Due to the frequency of the LFM signal varies with the time, we introduce the piecewise idea to solve the matching between the signal and the system parameters. To quantify the VR effect effectively, we propose a new spectrum amplification factor as the indicator. It is superior to another indicator, i.e. the crosscorrelated coefficient. The piecewise way, the re-scaled parameter used in the re-scaled VR method, the frequency reduced ratio used in the twice sampling VR method, and the LFM signal parameters on the VR effect are discussed, respectively. The results demonstrate that both the new spectrum amplification factor and the two proposed methods are effective in improving the LFM signal. Especially, the new spectrum amplification factor and the piecewise idea can also be used to process other kinds of frequency modulated signals whose spectrum have the determined form. As is well known, the frequency modulated signal widely exists in the engineering fields. For example, the speed of a rotary machine may be in a frequency modulated signal form. If we want to extract its fault feature in the vibration time series, we must process the weak frequency modulated signal. As a consequence, our method and results in the present work might have a referenced value in frequency modulated signal issues.

\section{Acknowledgments}

Jianhua Yang acknowledges financial support by the National Natural Science Foundation of China (grant no. 11672325), the Priority Academic Program Development of Jiangsu Higher Education Institutions, Top-notch Academic Programs Project of Jiangsu Higher Education Institutions. Miguel A. F. Sanjuán acknowledges the Spanish State Research Agency (AEI) and the European Regional Development Fund (FEDER) under project no. FIS2016-76883-P, and the jointly sponsored financial support by the Fulbright Program and the Spanish Ministry of Education (program no. FMECD-ST-2016).

\section{References}

[1] Landa, P.S., McClintock, P.V.E.: 'Vibrational resonance', J. Phys. A, 2000, 33, (45), pp. L433-L438

[2] Gammaitoni, L., Hänggi, P., Jung, P., et al.: 'Stochastic resonance', Rev. Mod. Phys., 1998, 70, (1), pp. 223-287
[3] Thomsen, J.J.: 'Some general effects of strong high-frequency excitation: stiffening, biasing and smoothening', J. Sound Vib., 2002, 253, (4), pp. $807-$ 831

[4] Thomsen, J.J.: 'Vibrations and stability: advanced theory, analysis, and tools' (Springer, Berlin, 2013)

[5] Blekhman, I.I.: 'Vibrational mechanics: nonlinear dynamic effects, general approach, applications' (World Scientific, Singapore, 2000)

[6] Yang, J.H., Liu, X.B.: 'Delay induces quasi-periodic vibrational resonance', $J$. Phys. A, 2010, 43, (12), p. 122001

[7] Yang, J.H., Liu, X.B.: 'Controlling vibrational resonance in a multistable system by time delay', Chaos, 2010, 20, (3), p. 033124

[8] Jeevarathinam, C., Rajasekar, S., Sanjuán, M.A.F.: 'Theory and numerics of vibrational resonance in Duffing oscillators with time-delayed feedback', Phys. Rev. E, 2011, 83, (6), p. 066205

[9] Yang, J.H., Zhu, H.: 'Vibrational resonance in Duffing systems with fractional-order damping', Chaos, 2012, 22, (1), p. 013112

[10] Yang, J.H., Sanjuán, M.A.F., Liu, H.G.: 'Enhancing the weak signal with arbitrary high-frequency by vibrational resonance in fractional-order Duffing oscillators', J. Comput. Nonlinear Dyn., 2017, 12, (2), p. 051011

[11] Yang, J.H.: 'Bifurcation and resonance in fractional-order systems' (Science Press, Beijing, 2017)

[12] Chizhevsky, V.N.: 'Experimental evidence of vibrational resonance in a multistable system', Phys. Rev. E, 2014, 89, (6), p. 062914

[13] Chizhevsky, V.N., Smeu, E., Giacomelli, G.: 'Experimental evidence of 'vibrational resonance' in an optical system', Phys. Rev. Lett., 2003, 91, (2), p. 220602

[14] Deng, B., Wang, J., Wei, X.: 'Effect of chemical synapse on vibrational resonance in coupled neurons', Chaos, 2009, 19, (1), p. 013117

[15] Deng, B., Wang, J., Wei, X., et al.: 'Vibrational resonance in neuron populations', Chaos, 2010, 20, (1), p. 013113

[16] Yu, H., Wang, J., Liu, C., et al.: 'Vibrational resonance in excitable neuronal systems', Chaos, 2011, 21, (4), p. 043101

[17] Bordet, M., Morfu, S.: 'Experimental and numerical enhancement of vibrational resonance in neural circuit', Electron. Lett., 2012, 48, (15), pp 903-905

[18] Uzuntarla, M., Yilmaz, E., Wagemakers, A., et al.: 'Vibrational resonance in a heterogeneous scale free network of neurons', Commun. Nonlinear Sci. Numer. Simul., 2015, 22, (1), pp. 367-374

[19] Jeevarathinam, C., Rajasekar, S., Sanjuán, M.A.F.: 'Vibrational resonance in groundwater-dependent plant ecosystems', Ecol. Complex, 2013, 15, (5), pp. $33-42$

[20] Roy-Layinde, T.O., Laoye, J.A., Popoola, O.O., et al.: 'Analysis of vibrational resonance in bi-harmonically driven plasma', Chaos, 2016, 26, (9), p. 093117

[21] Gosak, M., Perc, M., Kralj, S.: 'The impact of static disorder on vibrational resonance in a ferroelectric liquid crystal', Mol. Cryst. Liq. Cryst., 2012, 553, (1), pp. 13-20

[22] Liu, Y., Dai, Z., Lu, S., et al.: 'Enhanced bearing fault detection using stepvarying vibrational resonance based on Duffing oscillator nonlinear system', Shock Vib., 2017, 2017, (3), p. 5716296

[23] Chizhevsky, V.N., Giacomelli, G.: 'Vibrational resonance and the detection of aperiodic binary signals', Phys. Rev. E, 2008, 77, (1), p. 051126

[24] Elgamel, S.A., Soraghan, J.J.: 'Using EMD-FrFT filtering to mitigate very high power interference in chirp tracking radar', IEEE Signal Proc. Lett., 2011, 18, (4), pp. 263-266

[25] Kanno, A., Honda, S., Yamanaka, R., et al.: 'Ultrafast and broadband frequency chirp signal generation using a high-extinction-ratio optical modulator', Opt. Lett., 2010, 35, (24), pp. 4160-4162

[26] Kronauge, M., Rohling, H.: 'New chirp sequence radar waveform', IEEE Trans. Aerosp. Electron. Syst., 2014, 50, (4), pp. 2870-2877

[27] Zhu, F., Zhang, Q., Lei, Q., et al.: 'Reconstruction of moving target's HRRP using sparse frequency-stepped chirp signal', IEEE Sens. J., 2011, 11, (10), pp. $2327-2334$

[28] Michaels, J.E., Lee, S.J., Croxford, A.J., et al.: 'Chirp excitation of ultrasonic guided waves', Ultrasonics, 2013, 53, (1), pp. 265-270

[29] Song, J., Chang, J.H., Song, T.K., et al.: 'Coded tissue harmonic imaging with nonlinear chirp signals', Ultrasonics, 2011, 51, (4), pp. 516-521

[30] Dixon, R. C.: 'Spread spectrum systems: with commercial applications' (Wiley, New York, 1994)

[31] Lin, L., Wang, H., Lv, W.: 'Stochastic resonance system with linear random frequency fluctuation for aperiodic LFM signal', Nonlinear Dyn., 2017, 88, (2), pp. 1361-1371

[32] Lin, L., Wang, H., Lv, W., et al.: 'A novel parameter-induced stochastic resonance phenomena in fractional Fourier domain', Mech. Syst. Signal Process., 2016, 76, pp. 771-779

[33] Lin, L.F., Yu, L., Wang, H., et al.: 'Parameter-adjusted stochastic resonance system for the aperiodic echo chirp signal in optimal FrFT domain', Commun. Nonlinear Sci. Numer. Simul., 2017, 43, pp. 171-181

[34] Huang, D., Yang, J., Zhang, J., et al.: 'An improved adaptive stochastic resonance method for improving the efficiency of bearing faults diagnosis', Proc. Inst. Mech. Eng. C, J. Mech. Eng. Sci., 2018, 232, (13), pp. 2352-2368

[35] Jia, P.X., Wu, C.J., Yang, J.H., et al.: 'Improving the weak aperiodic signal by three kinds of vibrational resonance', Nonlinear Dyn., 2018, 91, (4), pp. 2699-2713

[36] Leng, Y.G., Wang, T.Y., Guo, Y., et al.: ‘Engineering signal processing based on bistable stochastic resonance', Mech. Syst. Signal Process., 2007, 21, (1) pp. $138-150$

[37] He, H.L., Wang, T.Y., Leng, Y.G., et al.: 'Study on non-linear filter characteristic and engineering application of cascaded bistable stochastic resonance system', Mech. Syst. Signal Process., 2007, 21, (7), pp. 2740-2749 